\title{
Cross-resistance to fluconazole induced by exposure to the agricultural azole tetraconazole: an environmental resistance school?
}

\author{
Marcos Fábio Gadelha Rocha, ${ }^{1,2}$ L. P. Alencar, ${ }^{2}$ M. A. N. Paiva, ${ }^{2}$ Luciana Magalhães Melo, ${ }^{2}$ \\ Silviane Praciano Bandeira, ${ }^{1}$ Y. B. Ponte, ${ }^{2}$ Jamille Alencar Sales, $^{2}$ G. M. M. Guedes, ${ }^{1}$ \\ D. S. C. M. Castelo-Branco, ${ }^{1}$ T. J .P. G. Bandeira, ${ }^{3}$ R. A. Cordeiro, ${ }^{1}$ W. A. Pereira-Neto, ${ }^{1}$ \\ G. S. Brandine, ${ }^{1}$ José Luciano Bezerra Moreira, ${ }^{1}$ José Júlio Costa Sidrim ${ }^{1}$ and Raimunda Sâmia \\ Nogueira Brilhante ${ }^{1}$
}

${ }^{1}$ Department of Pathology and Legal Medicine, Postgraduate Program in Medical Microbiology, Specialized Medical Mycology Center, Federal University of Ceará, Fortaleza, Ceará, Brazil, '2School of Veterinary Medicine, Postgraduate Program in Veterinary Sciences, State University of Ceará, Fortaleza, Ceará, Brazil and ${ }^{3}$ School of Medicine, Christus College - Unichristus, Fortaleza, Ceará, Brazil

\section{Summary}

\begin{abstract}
This study aimed to investigate the influence of tetraconazole and malathion, both used in agricultural activities, on resistance to fluconazole, itraconazole and voriconazole in Candida parapsilosis ATCC 22019. The susceptibility to tetraconazole, malathion, fluconazole, itraconazole and voriconazole, through broth microdilution. Then, 12 independent replicates, were separated and exposed to four treatment groups, each one containing three replicates: G1: tetraconazole; G2: malathion; G3: fluconazole (positive control); G4: negative control. Replicates from G1, G2 and G3, were exposed to weekly increasing concentrations of tetraconazole, malathion and fluconazole, respectively, ranging from MIC/2 to $32 \times \mathrm{MIC}$, throughout 7 weeks. The exposure to tetraconazole, but not malathion, decreased susceptibility to clinical azoles, especially fluconazole. The tetraconazole-induced fluconazole resistance is partially mediated by the increased activity of ATP-dependent efflux pumps, considering the increase in antifungal susceptibility after the addition of the efflux pump inhibitor, promethazine, and the increase in rhodamine 6G efflux and CDR gene expression in the G1 replicates. Moreover, MDR expression was only detected in G1 and G3 replicates, suggesting that MDR pumps are also involved in tetraconazoleinduced fluconazole resistance. It is noteworthy that tetraconazole and fluconazoletreated replicates behaved similarly, therefore, resistance to azoles of clinical use may be a consequence of using azoles in farming activities.
\end{abstract}

Key words: Candida sp., azole resistance, environmental resistance, tetraconazole, efflux pumps, CDR genes.

Correspondence: R. S. N. Brilhante, Department of Pathology and Legal Medicine, Postgraduate Program in Medical Microbiology, Specialized Medical Mycology Center, Federal University of Ceará, Fortaleza, Ceará, Brazil.

Tel.: 5585 3366-8319. Fax: 5585 3366-8303.

E-mail: brilhante@ufc.br

Submitted for publication 10 September 2015

Revised 20 November 2015

Accepted for publication 9 December 2015

\section{Introduction}

The overuse and misuse of antibacterial drugs are most likely responsible for the emergence of antimicrobial resistance in bacteria, especially in areas where resistant species are commonly isolated, such as hospital waste, ${ }^{1}$ untreated urban wastewater ${ }^{2}$ and livestock production and aquaculture effluent. ${ }^{3,4}$ Similarly, the detection of antifungal resistance is often associated with patients receiving prolonged antifungal 
treatment. However, antifungal resistance also affects microbial populations from aquatic and terrestrial environments, commonly involving hosts with no previous history of antifungal exposure. ${ }^{4-7}$

Although fluconazole resistance is more frequently reported, resistance to other azoles, such as ketoconazole, itraconazole and voriconazole, also occurs. ${ }^{4-7}$ This phenomenon is phenotypically stable, and in human strains, is the result of the action of several mechanisms, alone or combined. ${ }^{8-10}$ The increased expression of transmembrane transporters of the ATPbinding cassette family $(\mathrm{ABC})$ is described as the main mechanism for azole resistance in Candida spp. ABC efflux pumps generally have low substrate specificity, and produce energy through ATP hydrolysis to remove a wide variety of hydrophobic compounds from the cell, including azoles. ${ }^{11-13}$

In addition to the $\mathrm{ABC}$ pumps, the increased expression of the multidrug resistant (MDR) gene of the major facilitator superfamily, which encodes proton-dependent efflux pumps, is associated with specific fluconazole resistance. ${ }^{14}$ This gene is controlled by the transcription factor Mrrlp (multidrug resistant regulator), and overexpressed in resistant clinical strains. ${ }^{11,14-16}$

In addition, resistance may also be mediated by the increased expression of ERG11 gene, which leads to higher concentrations of the target enzyme Erg11p, ${ }^{17-}$ 19 hence, requiring greater amount of drug to inhibit fungal growth. ${ }^{11,20}$ Moreover, azole resistance may be mediated by mutations in ERG11 gene, decreasing the affinity between azoles and the enzyme Erg11p. ${ }^{17,18,20}$ Finally, azole resistance may also be associated with decreased activity of the enzyme $\Delta 5,6$-desaturase (Erg3p), encoded by ERG3 gene, leading to the decreased production of 14 $\alpha$-metilergosta-8,24(28)dien-3 $\beta, 6 \alpha$-diol, a toxic metabolite, hence promoting yeast survivability. ${ }^{11,21}$

The reports of azole resistance among Candida strains from animal and environmental sources with little or no contact with human hosts and no previous history of intentional antifungal exposure $e^{4,5,7,22,23}$ drew our attention, especially because this resistance occurs at higher rates, when compared to human strains. Based on the idea that resistance is established through the evolution of microorganisms in response to environmental stresses, ${ }^{24}$ it was hypothesised that these strains have developed azole resistance in response to the direct or indirect exposure to drugs used in agriculture. Thus, the antifungal susceptibility of one azole-susceptible standard Candida strain was assessed, after continuous exposure to compounds used in agriculture (one azole derivative and one organophosphate). Hence, the aim of this study was to investigate the contribution of tetraconazole and malathion for the in vitro development of azole resistance, using C. parapsilosis ATCC 22019 as a model strain.

\section{Materials and methods}

\section{Tested strain}

The strain C. parapsilosis ATCC 22019, which is susceptible to azoles of clinical use, was grown on potato dextrose agar for $48 \mathrm{~h}$, from one single colony. The susceptibility of this strain to the antifungal drugs and the tested compounds was determined, ${ }^{25}$ prior to beginning the experiments.

\section{Antifungal susceptibility testing}

The minimum inhibitory concentrations (MIC) of the antifungal drugs (fluconazole, itraconazole and voriconazole) and the tested agricultural compounds (tetraconazole and malathion) against one isolated colony of C. parapsilosis ATCC 22019, after growth on potato dextrose agar, were determined according to the document M27-A3, as recommended by the Clinical and Laboratory Standards Institute - CLSI. ${ }^{25}$ The susceptibility was investigated through the broth microdilution assay, in 96-well microplates, with a final volume of $200 \mu \mathrm{L}$ of RPMI buffered at $\mathrm{pH} 7$ with $0.165 \mathrm{~mol} \mathrm{l}^{-1}$ MOPS. The tested concentration ranges were $0.125-512 \mu \mathrm{g} \mathrm{ml}^{-1}$ for fluconazole (Zoltec, Pfizer, Brazil), $0.125-2048 \mu \mathrm{g} \mathrm{ml}^{-1}$ for tetraconazole (Domark 100 EC; Isagro SpA, Segrate, Milano, Italy) and malathion (Malathion 500 EC; De Sangosse Agrochemical, Curitiba, Paraná, Brazil) and 0.015-16 for itraconazole (Janssen Pharmaceutica, Beerse, Antwerp, Belgium) and voriconazole (Pfizer, New York, New York, USA). The plates were read after 24 and $48 \mathrm{~h}$ of incubation at $35^{\circ} \mathrm{C}$, but the MIC values considered were those obtained after $48 \mathrm{~h}$ of growth. MIC for azoles and malathion were the lowest drug concentration capable of inhibiting $50 \%$ of growth, when compared to the drug-free growth control. C. krusei ATCC 6258 and C. parapsilosis ATCC 22019 grown on potato dextrose agar were included as controls.

\section{Induction of resistance}

After performing the antifungal susceptibility assay, the following MIC values were obtained: $8,8,2$, 0.0625 and $0.03125 \mu \mathrm{g} \mathrm{ml}^{-1}$ for tetraconazole, 
malathion, fluconazole, itraconazole and voriconazole, respectively, against the strain C. parapsilosis ATCC 22019. It is important to highlight that the obtained clinical antifungal MICs were within the expected range for this control strain. ${ }^{25}$ Afterwards, this strain was subcultured into 12 tubes containing YEPD broth ( $0.5 \%$ yeast extract, $1 \%$ peptone and $2 \%$ dextrose). These tubes were, then, subjected to four different treatments, each one in three independent replicates: (i) tetraconazole (agricultural azole); (ii) malathion (agricultural organophosphate), (iii) fluconazole (positive control) and (iv) drug-free YEPD broth (negative control). The initial concentration to which the replicates of each treatment group were exposed was equivalent to half the MIC value of each treatment compound, i.e. 4,4 and $1 \mu \mathrm{g} \mathrm{ml}^{-1}$ for tetraconazole, malathion and fluconazole respectively. The three replicates of each treatment group were exposed to the same drug concentration for 1 week, during which these replicates were subcultured to YEPD broth containing the drugs, at a 48-h interval. At the end of each week, fluconazole susceptibility of all three independent replicates of each treatment group was assessed. Then, the concentration of each treatment drug was doubled and a new cycle of three subcultures was started. These procedures were performed until fluconazole MICs stabilised, which occurred when the independent replicates were exposed to drug concentrations 32 times higher than the initial MIC value of each tested drug. Additionally, at the end of the induction of resistance procedures, three independent replicates of each treatment group were stored at $4{ }^{\circ} \mathrm{C}$ in $10 \%$ glycerol for 10 months. After this period, the independent replicates were subcultured in drug-free YEPD broth, during 2 weeks, and the MICs for the antifungal drugs (fluconazole, itraconazole and voriconazole) and the agricultural drugs (tetraconazole and malathion) were reassessed. It is important to highlight that for the weekly evaluation of fluconazole susceptibility, the three replicates from the negative control treatment and C. krusei ATCC 6258 and C. parapsilosis ATCC 22019 grown on potato dextrose agar were included as controls. Moreover, all three independent replicates of each treatment group were tested in duplicate throughout this research.

\section{Investigation of resistance mechanisms}

Antifungal susceptibility with and without efflux pump inhibitor

Fluconazole, itraconazole and voriconazole MICs, with and without an efflux pump inhibitor, against the three independent replicates of the groups exposed to tetraconazole and fluconazole, and the drug-free group (negative control) were determined according to the document $\mathrm{M} 27-\mathrm{A} 3,{ }^{25}$ after finishing the protocol of inducing resistance, in order to verify the contribution of these pumps to the resistant phenotype. For such, MIC values were determined in the presence of a subinhibitory concentration (i.e. $32 \mu \mathrm{g} \mathrm{ml}^{-1}$ ) of promethazine, a CDR $1 p$ and CDR2p inhibitor. ${ }^{26}$ The obtained antifungal MICs before and after the addition of promethazine were observed. Malathion-treated group was not included in this analysis because this compound was not able to induce fluconazole resistance.

\section{Efflux of rhodamine $6 G$}

Since rhodamine 6G (R6G) acts as a substrate for CDR pumps, ${ }^{21,26}$ this fluorescent substance is used as a tool for the evaluation of their efflux activity. Here, the test was performed according to the protocol described by Ivnitski-Steele et al. [26] and Vale-Silva et al. [21] with modifications. Briefly, each of the three independent replicates of the groups exposed to tetraconazole and fluconazole, and the drug-free group (negative control) were grown in triplicate (nine replicates for each treatment group), overnight in $5 \mathrm{ml}$ of YEPD broth at $37{ }^{\circ} \mathrm{C}$ for $24 \mathrm{~h}$. Afterwards, the replicates were transferred to $5 \mathrm{ml}$ of fresh YEPD broth at $37^{\circ} \mathrm{C}$, under constant agitation, allowing growth until reaching a concentration $2 \times 10^{7}$ cells ml$^{-1}$. Cells were centrifuged at $4500 \mathrm{~g}$ for $5 \mathrm{~min}$, the supernatant was discarded, and the pellet was washed twice in $2 \mathrm{ml}$ of PBS ( $\mathrm{pH}$ 7.0). Subsequently, cells were deprived of energy by incubation under constant agitation in $2 \mathrm{ml}$ of PBS at $37^{\circ} \mathrm{C}$, for $1 \mathrm{~h}$. Then, R6G was added to obtain a final concentration of $15 \mu \mathrm{mol} \mathrm{l}^{-1}$ and the suspension was incubated at $37^{\circ} \mathrm{C}$, for $1 \mathrm{~h}$, in the dark, under constant agitation, in order to allow the influx of R6G into the yeast cells.

After incubation, the cells were washed twice in $2 \mathrm{ml}$ of PBS, at $4{ }^{\circ} \mathrm{C}$, and suspended in $300 \mu \mathrm{L}$ of PBS. Then, $12.5 \mu \mathrm{L}$ of the suspension were added to $32.5 \mu \mathrm{l}$ of PBS in a 96-well plate (Hard-Shell ${ }^{\circledR}$ LowProfile Thin-Wall) and left to settle for $5 \mathrm{~min}$. Subsequently, glucose was added to reach a final concentration of $1 \%$ and the relative fluorescence units (RFU) were recorded at $1 \mathrm{~min}$ intervals, during $60 \mathrm{~min}$ at $37^{\circ} \mathrm{C}^{27}$ The fluorescent signal was measured with a CFX96 Touch $^{\mathrm{TM}}$ Real-Time PCR Detection System (Bio Rad, Hercules, California, USA), using 610-650 nm filtre. All replicates of the different treatment groups were read in the presence and in the 
absence of glucose. Negative controls were prepared with PBS instead of glucose. Replicates of the malathion-treated group were not included in the R6G assay because this compound did not induce fluconazole resistance.

\section{Ergosterol content}

The amount of cellular sterols within the treated yeast cells was quantified, in order to detect phenotypical alterations in ergosterol biosynthesis that could lead to azole resistance. For such, the extraction of total sterols was carried out, as described by Cordeiro et al. [28] The three independent replicates of each treatment group were grown overnight in YEPD broth at $37{ }^{\circ} \mathrm{C}$ and centrifuged at $9660 \mathrm{~g}$ for $3 \mathrm{~min}$. Pellets were washed in PBS and the turbidity was adjusted to 0.5 on McFarland scale (i.e. approximately 1.0$5.0 \times 10^{6}$ cells ml${ }^{-1}$ ). Then, $1 \mathrm{ml}$ of this suspension was centrifuged at 9,660 $\times g$ for $3 \mathrm{~min}$ and the pellet was resuspended in $0.5 \mathrm{ml}$ of $\mathrm{KOH}$ alcoholic solution $\left(0.7 \mathrm{~mol} \mathrm{l}^{-1} \mathrm{KOH}\right.$ in $60 \%$ ethanol) and incubated for $1 \mathrm{~h}$ at $95{ }^{\circ} \mathrm{C}$, in a water bath. After cooling, $0.6 \mathrm{ml}$ of $\mathrm{n}$-hexane was added to the tubes, which were vigorously vortexed for $5 \mathrm{~min}$. Then, the tubes were centrifuged at $13,416 \times g$ for $1 \mathrm{~min}$ and the top organic layer was transferred to a new tube and mixed with $1 \mathrm{ml}$ of $\mathrm{n}$-hexane. The absorbance of this solution was read at $295 \mathrm{~nm}$, in a spectrophotometer. The optical density data were compared to those obtained in a standard curve with ergosterol (SigmaAldrich, St. Louis, Missouri, USA). Readings were performed in duplicate for each of the three independent replicates of the treatment groups. The data obtained from replicates exposed to tetraconazole and fluconazole were compared to the negative control. Malathion-treated replicates were not included in this analysis.

\section{CDR, MDR and ERG11 gene expression}

The expression of CDR and MDR (efflux pump genes) and ERG11 (gene that encodes 14-alpha-demethylase, the target enzyme for azoles) was also assessed in treated groups, since the overexpression of these genes is the most common mechanism of azole resistance. For such, total RNA was extracted, in triplicate, from each of the three independent replicates of the groups exposed to tetraconazole and fluconazole and the drug-free group (negative control), using the RNeasy mini kit (Qiagen, GmbH, Hilden, Germany) according to the manufacturer's instructions. Briefly, the independent replicates were grown in YEPD broth at $37^{\circ} \mathrm{C}$, for $24 \mathrm{~h}$, until reaching the mid-log growth phase. Then, $1 \mathrm{ml}$ of each independent replicate, containing approximately $5 \times 10^{7}$ cells was centrifuged at $4500 \boldsymbol{g}$ and the pellet was lysed with RLT buffer and $0.45-0.55 \mathrm{~mm}$ glass beads, using Precellys 24 disrupter (Bertin Technologies, Montigny le Bretonneux, Yvelines, France). The lysate was diluted $1: 1$ with $70 \%$ ethanol and transferred to a spin column. Genomic DNA was degraded using RNase-free DNase for $15 \mathrm{~min}$ at room temperature. After three washes, the RNA was eluted in RNase-free water and the concentration was measured using Qubit fluorometer and RNA Assay Kit (Invitrogen, Paisley, UK). Reverse transcription was performed with $1 \mu \mathrm{g}$ of RNA combined with $1 \mu \mathrm{L}$ of Improm II (Promega, Madison, Wisconsin, USA), $0.5 \mathrm{mmol} \mathrm{l}^{-1}$ of each dNTP, $40 \mathrm{U}$ of RNaseOUT, $0.5 \mu \mathrm{g}$ of random primers and RNase-free water to a final volume of $20 \mu \mathrm{L}$. Reverse transcription was performed at $42{ }^{\circ} \mathrm{C}$, for $60 \mathrm{~min}$, followed by $70{ }^{\circ} \mathrm{C}$, for $15 \mathrm{~min}$. The first strand cDNA products were stored at $-80{ }^{\circ} \mathrm{C}$ for later use as templates for quantitative real-time PCR (qPCR). Negative controls and RT blanks were prepared under the same conditions, but without inclusion of RNA or reverse transcriptase respectively.

Expression levels of CDR, MDR and ERG11 and reference endogenous $A C T 1$ and $18 S$ genes were assessed through qPCR. The reaction mix contained $0.5 \mu \mathrm{L}$ of cDNA, $1.5 \mu \mathrm{L}$ of primer $\left(2 \mu \mathrm{mol} \mathrm{l}^{-1}\right)$ and $7.5 \mu \mathrm{L}$ of $2 \times$ FastStart Universal SYBR Green Master (Roche, Indianapolis, IN, USA), in a final volume of $15 \mu \mathrm{L}$ per reaction. The reactions were carried out in a CFX96 Touch $^{\text {TM }}$ Real-Time PCR Detection System (Bio Rad) with the following amplification conditions: enzyme activation at $95{ }^{\circ} \mathrm{C}$ for $5 \mathrm{~min}$, followed by 40 cycles, each consisting of denaturation at $95{ }^{\circ} \mathrm{C}$ for $30 \mathrm{sec}$, annealing at a specific temperature for each primer pair for $30 \mathrm{sec}$ (Table S1) and extension at $72{ }^{\circ} \mathrm{C}$ for $30 \mathrm{s.}^{29}$ Fluorescence data were acquired at the end of each extension step. After the final extension, the specificity was ascertained by melting of the amplicons, detecting the fluorescence obtained at the annealing temperature of each primer, at an increase of $0.5^{\circ} \mathrm{C}$ every $5 \mathrm{~s}$, until the temperature reached $95^{\circ} \mathrm{C}$. To determine the linearity and reaction efficiency for each primer, the standard curve was plotted using serial dilutions of cDNA with qPCR conditions identical to those established by the experimental protocol. Negative control and RT blank were used. The relative quantification of the gene expression was performed using the $2^{-\Delta \Delta \mathrm{Cq}}$ method. ${ }^{30}$ Target gene expression was normalised against ACT1 and $18 \mathrm{~S}$ mean expression. Overexpression occurred when the 
levels obtained for the target genes from the replicates of the treated groups (tetraconazole or fluconazole) were statistically higher than those obtained from the negative group. Threshold and $\mathrm{Cq}$ (threshold cycle) values were automatically determined by the BioRad CFX manager 3.0 software (BioRad), using default parameters. Replicates of the malathion-treated group were not included in this analysis.

\section{Statistical analysis}

The correlation between the exposure to each treatment group and fluconazole MICs was compared by using Pearson's correlation coefficient $\left(r^{2}\right)$. The efflux pump activity of different groups was compared through the analysis of R6G efflux with and without glucose, using the Kruskal-Wallis test with Dunn's multiple comparison test for post hoc comparisons. The gene expression levels were compared using one-way analysis of variance (ANOVA), with Bonferroni's post hoc test for comparisons. In all tests, $P<0.05$ was considered statistically significant.

\section{Results}

\section{Induction of resistance}

The three independent replicates of $C$. parapsilosis ATCC 22019 of each treatment group, exposed to increasing concentrations of tetraconazole, malathion and fluconazole (positive control) evolved to present higher tolerance to the compound they were exposed. At the end of the experiment, after storage in 10\% glycerol, at $4{ }^{\circ} \mathrm{C}$, for 10 months, the replicates were grown in drug-free YEPD broth, for 2 weeks, and reassessed for their susceptibility to the compounds to which they were previously exposed. All tested compounds (tetraconazole, malathion and fluconazole) presented increased MICs against the set of replicates exposed to the respective compound. Tetraconazole MICs increased from 4 to 8 times, malathion MICs increased from 128 to 512 times, and fluconazole MICs increased from 64 to 128 .

In the tetraconazole-treated group, a positive correlation $\left(r^{2}=0.97\right)$ between the exposure concentration of tetraconazole and fluconazole MICs was observed for all the three independent replicates. Resistance to this clinical azole was rapidly induced by exposure to increasing concentrations of the agricultural drug. In fact, fluconazole resistance started after exposure to tetraconazole concentrations of $2 \times$ MIC (Fig. 1). Similar results were observed for the replicates that were

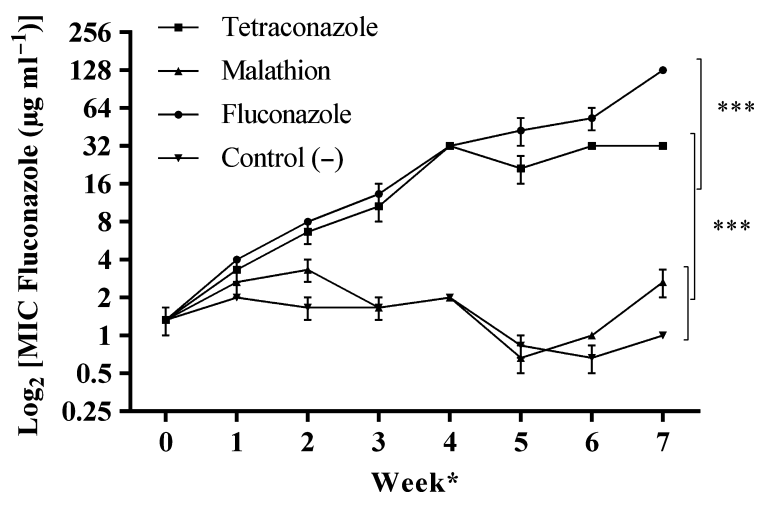

Figure 1 Dynamics of fluconazole susceptibility of independent replicates of C. parapsilosis ATCC 22019 exposed to increasing concentrations of tetraconazole $(n=3)$, malathion $(n=3)$ and fluconazole $(n=3)$. Data are expressed as geometric mean \pm standard deviation (SD) of each treatment group. Unexposed group: control $(-)(n=3)$. '0' represents the beginning of treatment, with the MICs obtained prior to resistance induction assay. *Drug concentration was doubled weekly and fluconazole susceptibility was tested at the end of each week. Data obtained for each treatment group were compared to drug-free group (negative control). ${ }^{* * *} P<0.001$. In the $Y$-axis, antifungal concentration ranges from $0.5 \mu \mathrm{g} \mathrm{ml}^{-1}\left[\log _{2}(0.5)=-1\right]$ to $256 \mu \mathrm{g} \mathrm{ml}^{-1}$ $\left[\log _{2}(256)=8\right]$.

exposed to fluconazole (positive control). On the other hand, susceptibility to fluconazole did not change in the three replicates exposed to malathion or the negative control replicates. Therefore, the malathion-treated group was not included in the subsequent assays, since exposure to this compound did not interfere with fluconazole MICs.

\section{Antifungal susceptibility testing with and without efflux pump inhibitor}

After the induction of resistance, the independent replicates of the groups exposed to tetraconazole and fluconazole, and the drug-free group (negative control) were evaluated for their susceptibility to antifungal agents used in medical practice. In addition to fluconazole resistance, both itraconazole and voriconazole presented increased MIC values against the three replicates exposed to tetraconazole, when compared to the negative control, and similar results were obtained for the positive control drug (fluconazole) (Fig. 2 and Table S2).

The antifungal susceptibility testing showed that efflux pump inhibition in the three tetraconazole replicates promoted 3-dilution and 4-dilution decrease $(P<0.001)$, in MIC for itraconazole and voriconazole respectively (Fig. 2). However, even though CDR efflux 


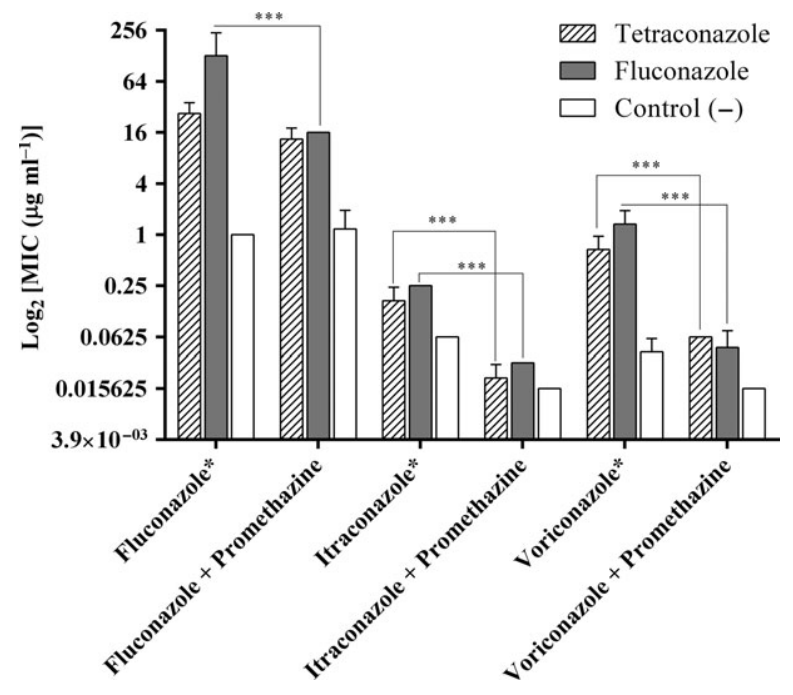

Figure 2 Antifungal susceptibility of the three independent replicates of tetraconazole-treated and fluconazole-treated groups and negative control group, with and without efflux pump inhibitor, after inducing antifungal resistance. Data are expressed as geometric mean \pm SD of each treatment group. Unexposed group: Control (-). Data are compared between replicates of the same treatment group, with and without the addition of promethazine. *Indicates a significant increase $(P<0.05)$ in MIC values of fluconazole, itraconazole and voriconazole, after exposure of replicates to increasing concentrations of tetraconazole $(n=3)$ and fluconazole $(n=3)$ compared to the unexposed group $(n=3)$. $* * * P<0.001$. In the $Y$-axis, antifungal concentration ranges from $0.015 \mu \mathrm{g} \mathrm{ml}^{-1}\left[\log _{2}(0.015)=-6\right]$ to $256 \mu \mathrm{g} \mathrm{ml}^{-1}$ $\left[\log _{2}(256)=8\right]$.

pump inhibition with promethazine caused a 1-dilution decrease in fluconazole MICs against the three tetraconazole-treated replicates, the resulting MIC values were not statistically different from the fluconazole MICs obtained without promethazine. This finding demonstrates that other mechanisms contribute to fluconazole resistance in the three tetraconazole-treated independent replicates. Similar results were also observed for the three fluconazole-treated replicates (positive control), against which the three tested azole drugs (fluconazole, itraconazole and voriconazole) also presented decreased $(P<0.001)$ MIC values when promethazine was added (Fig. 2).

\section{Efflux of rhodamine 6G}

The three tetraconazole-independent replicates presented increased efflux of R6G, when glucose was added to give the energetic support for the activity of CDR efflux pumps. The efflux of R6G in these replicates was similar to that observed in the fluconazole-treated replicates (positive control), demonstrating the increased activity of the energy-dependent efflux pumps (Fig. 3).

\section{Ergosterol content}

The ergosterol concentrations (mean $\pm \mathrm{SE}$ ) extracted from the three independent replicates of the groups exposed to tetraconazole and fluconazole, and the drug-free group (negative control) were $4.13 \pm 0.67$, $3.83 \pm 0.35$ and $3.58 \pm 0.46 \mu \mathrm{mol} \mathrm{l}^{-1}$ respectively. Thus, previous exposure to agricultural or clinical azole was not able to interfere with the ergosterol concentration of yeast cells.

\section{$C D R, M D R$ and ERG11 gene expression}

In the three tetraconazole-treated replicates, the expression of the CDR gene was increased, while the expression of ERG11 gene was not altered, when compared to the negative control. These results were similar to those found for the three fluconazole-treated
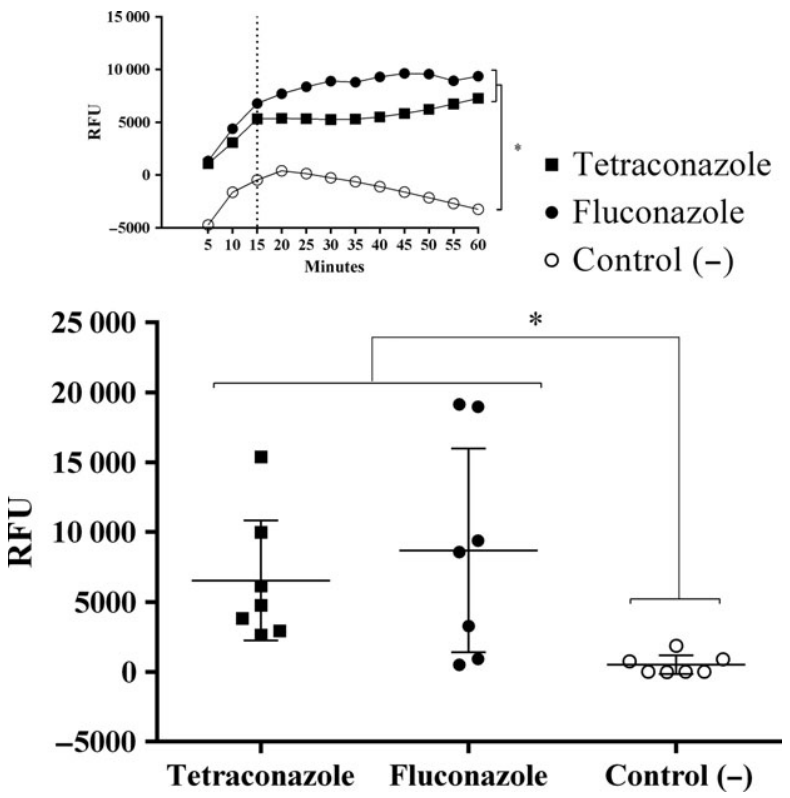

Figure 3 Efflux of rhodamine 6G by the three independent replicates of tetraconazole-treated and fluconazole-treated groups and negative control group, after induction of antifungal resistance. Data are expressed as relative fluorescence units (RFU). The top graph shows the RFU curve of rhodamine $6 \mathrm{G}$ efflux, demonstrating a plateau starting at $15 \mathrm{~min}$ after the addition of glucose. Each point on the curve represents the mean RFU of replicates with glucose minus the mean RFU of replicates without glucose. The lower graph depicts the median with interquartile range of the difference in RFU of the replicates with and without glucose, 15 min after the addition of glucose. ${ }^{*} P<0.05$. 
replicates (Fig. 4). The MDR 1 gene reached detectable levels only in the replicates exposed to tetraconazole and fluconazole, but not in those submitted to the negative control. Thus, MDR1 expression level was not calculated. It is important to highlight that neither primer-dimers nor unspecific products were detected in qPCR amplifications.

\section{Discussion}

Candida parapsilosis ATCC 22019 was chosen due to its phenotypical stability, as it is a quality control strain for antifungal susceptibility testing, according to the recommendations of CLSI, which were followed during the present research. ${ }^{25}$ In addition, this particular strain was originally recovered from a human case of sprue and the species C. parapsilosis is commonly isolated from the microbiota of humans and other animals and from terrestrial and aquatic environmental sources, often presenting reduced azole susceptibility. ${ }^{4,5,7,31}$ These characteristics supported the choice of this strain as a model for inducing antifungal resistance through the chronic exposure to compounds used in agricultural practices.

The effect of the in vitro exposure of an azole-susceptible Candida strain to the azole tetraconazole and the organophosphate malathion, both widely used in farming activities, and its consequence on the susceptibility of microorganisms to azoles of clinical use were evaluated. The obtained results indicate that exposure of $C$. parapsilosis to tetraconazole decreases susceptibility to fluconazole, itraconazole and voriconazole, similar to what was seen in the positive control group exposed to fluconazole. However, exposure to malathion did not change the susceptibility to the tested antifungal drugs; hence, the three malathion- treated independent replicates were not included in the analyses to investigate the resistance mechanisms.

The stress caused by tetraconazole exposure, as demonstrated in this study, most likely promotes cellular mechanisms to escape from the effects of this agricultural antifungal. This allows the development of important characteristics that also promote fungal protection against antifungal agents with similar mechanisms of action. The induction of azole resistance in Candida species has previously been observed due to in vitro ${ }^{18,32-34}$ and in vivo ${ }^{35}$ continuous exposure to azoles of medical use. In some researches, the MIC values of several azole drugs were simultaneously increased and not only the MIC of the drug used in the induction assay. ${ }^{33,34}$ Similarly, in this study, the MIC values of fluconazole, itraconazole and voriconazole were significantly increased, after the continuous in vitro exposure of three C. parapsilosis ATCC 22019 independent replicates to increasing concentrations of tetraconazole. These results are equivalent to those obtained for the positive control drug (fluconazole), demonstrating that exposure to clinical azole, ${ }^{18,32-35}$ as well as to agricultural drugs, may be responsible for the development of cross-resistance to several azoles in strains of Candida spp. and other fungal species. ${ }^{36-38}$

There are several reports of resistance in fungal species, which involves common mechanisms, regardless of the triggering factor, i.e. azoles used in human and animal medical practice, or in agricultural practice. These indications associated with the recovery of resistant fungal strains from the microbiota of animals without prior history of antifungal treatment ${ }^{4,5,7}$ reinforce the assumption of the existence of a selective pressure for the development of antifungal-resistant strains within the rural and/or wild environment. This environmental adaptation is crucial for the
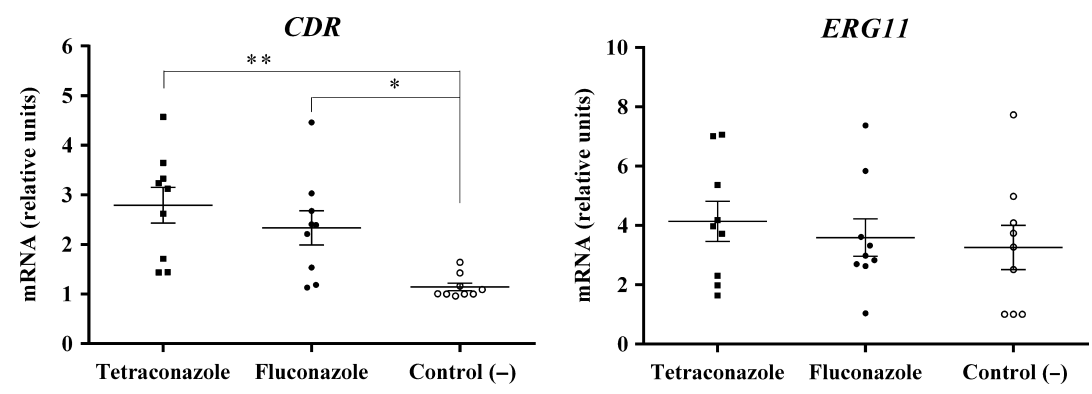

Figure 4 Expression of CDR and ERG11 genes in the three independent replicates of tetraconazole-treated and fluconazole-treated groups and negative control group, after induction of antifungal resistance. Data are normalised to mean signal of ACT1 and $18 S$ genes. The expression of one replicate of the negative control group was used as calibrator and their transcription level was set as ' 1 relative unit'. Data are expressed as dispersion and mean $\pm \mathrm{SE}$ of the replicates. Data of each treatment are compared to the negative control group. ${ }^{*} P<0.05 ;{ }^{* *} P<0.01$. 
development of commensal and pathogenic characteristics of Candida spp. ${ }^{39}$ In this sense, Hube [39] proposed the existence of 'commensal virulence schools' in which the microorganism develops certain characteristics to adapt or successfully infect the host. Similarly, the stress caused by the exposure to azoles in agriculture $^{36}$ (e.g. tetraconazole) could be an 'environmental resistance school', promoting the development of important features in microorganisms to enhance their survivability in the environment. Finally, the development of these features seems to induce crossresistance to medical azoles.

The knowledge on azole resistance in C. parapsilosis is still incipient. Even though this phenomenon probably results from a combination of classical mechanisms studied in other Candida species, ${ }^{14,35,40,41}$ the high MIC values of azoles against $C$. parapsilosis may also be associated with other molecular mechanisms that still need to be elucidated. ${ }^{40}$ However, it has been show that in fluconazole-resistant $C$. parapsilosis, $C D R$ overexpression is more common than the overexpression of MDR 1 , and both are more common than overexpression of ERG11. ${ }^{14,35,40,41}$ Thus, in this study, we primarily decided to investigate the drug-induced efflux-mediated mechanisms of resistance in C. parapsilosis. In this context, it was observed that tetraconazole exposure increased the activity of efflux pumps in C. parapsilosis, suggesting that this induced resistance is mainly associated with increased drug efflux through ATP-dependent pumps. These results are similar to those obtained for the fluconazole-treated (positive control) replicates in this study, and in others. ${ }^{18,32-34,40,41}$ One of the evidences for the involvement of ATP-dependent pumps is the reduction of the antifungal MICs, especially those of itraconazole and voriconazole, against the azoleresistant tetraconazole-treated replicates, when these efflux pumps are inhibited by promethazine. ${ }^{42}$ Other evidence for the involvement of these pumps in the development of antifungal resistance after exposure to tetraconazole is the increase in R6G efflux, which presented clonal difference between the independent replicates, based on the observed efflux outliers and the relative stability in efflux activity among the replicates from the negative control group. These findings demonstrated that the CDR efflux pumps, among other ATP-dependent pumps, are primarily involved in the development of azole resistance. Moreover, this observation was confirmed by the increased CDR gene expression in tetraconazole-treated replicates, similar to what was seen for the fluconazole-treated replicates.

The inhibition of the CDR efflux pumps reduced the azole MICs against the tetraconazole and fluconazole- treated replicates, but these MICs were still higher than those obtained against the negative control group. Moreover, CDR efflux pump inhibition did not cause significant reduction in fluconazole MICs against tetraconazole-treated replicates, which suggests the involvement of MDR proton pumps in this azoleinduced resistance. ${ }^{14,43}$ Additionally, the detection of MDR1 transcripts only in tetraconazole and fluconazole-treated replicates, but not in negative control, strengthens the assumption that these proton pumps are also involved in the development of tetraconazoleinduced fluconazole resistance, ${ }^{43}$ similar to what has been described for clinical strains. ${ }^{14,35,40}$ The maintenance of the amount of ergosterol extracted from the tetraconazole and fluconazole-treated C. parapsilosis ATCC 22019 replicates, when compared to the negative control, and the undifferentiated ERG11 expression between treated replicates and the negative control, showed that, in this study, increased ergosterol biosynthesis was not involved with the development of resistance. ${ }^{43}$ However, mutation in genes involved in ergosterol biosynthesis cannot be ruled out, especially, considering the possibility that more than one resistance mechanism may be acting at the same time. Even though we did not perform a sequence analysis of the genes involved in the biosynthesis of ergosterol, it is known that the single-nucleotide polymorphism (SNP) Y132F in ERG11 nucleotide sequence is the most common alteration in strains of fluconazole-resistant C. parapsilosis. ${ }^{14,40}$

The investigation of the possible resistance mechanisms demonstrated that the tetraconazole-treated replicates presented similar results to those treated with fluconazole. Thus, we have demonstrated that resistance to medical azoles may be a consequence of the exposure to agricultural azoles. In C. parapsilosis ATCC 22019, for instance, this phenomenon seems to be mainly associated with the increased activity of CDR efflux pumps, as a response to the continuous exposure to a drug-rich environment. These adaptive changes decrease azole susceptibility through unspecific pathways and seem to be phenotypically stable, after the storage of the strains at $4{ }^{\circ} \mathrm{C}$ for 10 months in glycerol. Thus, we believe that the misuse or prolonged use of azoles in humans ${ }^{8,9,16}$ and also in the environment may be the cause of resistance to these drugs in strains from diverse origins. The selection of Candida strains with reduced antifungal susceptibility in environments of agricultural production is relevant because these strains may colonise cultivated food items, and, subsequently, they may colonise the gastrointestinal tract of humans and other animals. In 
addition, considering that these agricultural drugs have long residual effect, ${ }^{44}$ their direct action on the yeast microbiota of humans and animals that feed on products with drug residues may also be speculated. Either or both mechanisms may be involved in the recovery of azole resistant Candida strains from several animal species, such as prawns, porcupine, raptors, rheas and tortoises, as reported in previous works of our group. ${ }^{4,5,7,45,46}$

The occurrence of azole resistance in strains from veterinary and environmental sources suggests that these strains can acquire such features in the environment, ${ }^{36,38,47,48}$ as a response to stress. ${ }^{24}$ Thus, in allusion to what was described by Hube [39] who defended the existence of 'commensal virulence schools', the presence of agricultural azoles in the environment would function as an 'environmental resistance school', and, in this case, tetraconazole would act as an intensive school, since it acts on environmental microbial communities for periods longer than 90 days. ${ }^{44}$ Thus, these data bring perspectives for further studies on the impact of drugs and other chemical compounds used in agriculture on microbial populations and on the induction of azole resistance in the environment.

\section{Acknowledgments}

This work was supported by grants from the National Council for Scientific and Technological Development (CNPq; Brazil; 307606/2013-9; 443167/2014-1; PNPD-UFC/Microbiologia Médica/CAPES).

\section{References}

1 Varela AR, Ferro G, Vredenburg J et al. Vancomycin resistant enterococci: from the hospital effluent to the urban wastewater treatment plant. Sci Total Environ 2013; 450-451: 155-61.

2 Birošová L, Mackul'ak T, Bodík I et al. Pilot study of seasonal occurrence and distribution of antibiotics and drug resistant bacteria in wastewater treatment plants in Slovakia. Sci Total Environ 2014; 490: 440-4.

3 Rebouças RH, de Sousa OV, Lima AS et al. Antimicrobial resistance profile of Vibrio species isolated from marine shrimp farming environments (Litopenaeus vannamei) at Ceará, Brazil. Environ Res 2011; 111: 21-24.

4 Brilhante RSN, Paiva MAN, Sampaio CMS et al. Yeasts from Macrobrachium amazonicum: a focus on antifungal susceptibility and virulence factors of Candida spp. FEMS Microbiol Ecol 2011; 76: 268-77.

5 Castelo-Branco DSCM, Brilhante RSN, Paiva MAN et al. Azoleresistant Candida albicans from a wild Brazilian porcupine (Coendou prehensilis): a sign of an environmental imbalance? Med Mycol 2013; 51: 555-60.

6 Cordeiro RA, Teixeira CEC, Brilhante RSN et al. Minimum inhibitory concentrations of amphotericin B, azoles and caspofungin against Candida species are reduced by farnesol. Med Mycol 2013; 51: 53-9.
7 Brilhante RSN, Alencar LP, Cordeiro RA et al. Detection of Candida species resistant to azoles in the microbiota of rheas (Rhea americana): possible implications for human and animal health. J Med Microbiol 2013; 62: 889-95.

8 White TC, Holleman S, Dy F et al. Resistance mechanisms in clinical isolates of Candida albicans. Antimicrob Agents Chemother 2002; 46: 1704-13.

9 Lopez-Ribot JL, McAtee RK, Lee LN et al. Distinct patterns of gene expression associated with development of fluconazole resistance in serial candida albicans isolates from human immunodeficiency virus-infected patients with oropharyngeal candidiasis. Antimicrob Agents Chemother 1998; 42: 2932-7.

10 Cannon RD, Lamping E, Holmes AR et al. Efflux-mediated antifungal drug resistance. Clin Microbiol Rev 2009; 22: 291-321.

11 Akins RA. An update on antifungal targets and mechanisms of resistance in Candida albicans. Med Mycol 2005; 43: 285-318.

12 Tsao S, Rahkhoodaee F, Raymond M. Relative contributions of the Candida albicans $\mathrm{ABC}$ transporters $\mathrm{Cdr} 1 \mathrm{p}$ and $\mathrm{Cdr} 2 \mathrm{p}$ to clinical azole resistance. Antimicrob Agents Chemother 2009; 53: 1344-52.

13 Coste AT, Turner V, Ischer $\mathrm{F}$ et al. A mutation in Taclp, a transcription factor regulating CDR1 and CDR2, is coupled with loss of heterozygosity at chromosome 5 to mediate antifungal resistance in Candida albicans. Genetics 2006; 172: 2139-56.

14 Grossman NT, Pham CD, Cleveland AA et al. Molecular mechanisms of fluconazole resistance in Candida parapsilosis Isolates from a U.S. Surveillance System. Antimicrob Agents Chemother 2015; 59: 1030-7.

15 Lohberger A, Coste A, Sanglard D. Distinct roles of Candida albicans drug resistance transcription factors TAC1, MRR1, and UPC2 in virulence. Eukaryot Cell 2014; 13: 127-42.

16 Morschhäuser J, Barker KS, Liu TT et al. The transcription factor Mrr1p controls expression of the MDR1 efflux pump and mediates multidrug resistance in Candida albicans. PLoS Pathog 2007; 3: 1603-16.

17 Xie JL, Polvi EJ, Shekhar-Guturja T et al. Elucidating drug resistance in human fungal pathogens. Future Microbiol 2014; 9: 523-42.

18 Marr KA, Lyons CN, Ha K et al. Inducible azole resistance associated with a heterogeneous phenotype in Candida albicans. Antimicrob Agents Chemother 2001; 45: 52-9.

19 Flowers SA, Barker KS, Berkow EL et al. Gain-of-function mutations in UPC2 are a frequent cause of ERG11 upregulation in azole-resistant clinical isolates of Candida albicans. Eukaryot Cell 2012; 11: 1289-99.

20 Xiang M-J, Liu J-Y, Ni P-H et al. Erg11 mutations associated with azole resistance in clinical isolates of Candida albicans. FEMS Yeast Res 2013; 13: 386-93.

21 Vale-Silva LA, Coste AT, Ischer F et al. Azole resistance by loss of function of the sterol $\Delta 5,6$ - desaturase gene (ERG3) in Candida albicans does not necessarily decrease virulence. Antimicrob Agents Chemother 2012; 56: 1960-8.

22 Jin Y, Lin D. Fungal urinary tract infections in the dog and cat: a retrospective study (2001-2004). J Am Anim Hosp Assoc 2005; 41: 373-81.

23 Nawange SR, Singh K, Naidu J et al. Naturally acquired systemic dual infection caused by Candida famata (Debaryomyces hansenii) and Candida catenulata in albino rats bred for sale in the market at Jabalpur (Madhya Pradesh), India. Mycoses 2010; 53: 173-5.

24 Cannon RD, Lamping E, Holmes AR et al. Candida albicans drug resistance - Another way to cope with stress. Microbiology 2007; 153: 3211-7.

25 CLSI. Reference Method for Broth Dilution Antifungal Susceptibility Testing of Yeasts. Approved Standard M27-A3, 3rd edn. Wayne, PA, 2008.

26 Ivnitski-Steele I, Holmes AR, Lamping E et al. Identification of Nile red as a fluorescent substrate of the Candida albicans ATP-binding cassette transporters $\mathrm{Cdr} 1 \mathrm{p}$ and $\mathrm{Cdr} 2 \mathrm{p}$ and the major facilitator superfamily transporter Mdr1p. Anal Biochem 2009; 394: 87-91.

27 Silva LV, Sanguinetti M, Vandeputte P et al. Milbemycins: more than efflux inhibitors for fungal pathogens. Antimicrob Agents Chemother 2013; 57: 873-86. 
28 Cordeiro RA, Marques FJF, Cordeiro RA et al. Synthesis and antifungal activity in vitro of isoniazid derivatives against Histoplasma capsulatum var. capsulatum. Antimicrob Agents Chemother 2014; 58: 2504 11.

29 Pulcrano G, Panellis D, De Domenico G et al. Ambroxol influences voriconazole resistance of Candida parapsilosis biofilm. FEMS Yeast Res 2012; 12: 430-8.

30 Livak KJ, Schmittgen TD. Analysis of relative gene expression data using real-time quantitative PCR and the 2(-Delta Delta C(T)) method. Methods 2001; 25: 402-8.

31 Brilhante RSN, Paiva MAN, Sampaio CMS et al. Surveillance of azole resistance among Candida spp. as a strategy for the indirect monitoring of freshwater environments. Water Air Soil Pollut 2015; 226: 52.

32 Silva CR, Andrade Neto JB, Sidrim JJC et al. Synergistic effects of amiodarone and fluconazole on Candida tropicalis resistant to fluconazole. Antimicrob Agents Chemother 2013; 57: 1691-700.

33 Barchiesi F, Calabrese D, Falconi L et al. Experimental induction of fluconazole resistance in Candida tropicalis ATCC 750. Antimicrob Agents Chemother 2000; 44: 1578-84.

34 Pinto e Silva AT, Costa-De-Oliveira S, Silva-Dias A et al. Dynamics of in vitro acquisition of resistance by Candida parapsilosis to different azoles. FEMS Yeast Res 2009; 9: 626-33.

35 Zhang L, Xiao M, Watts MR et al. Development of fluconazole resistance in a series of Candida parapsilosis isolates from a persistent candidemia patient with prolonged antifungal therapy. BMC Infect Dis 2015; 15: 340.

36 Müller F-MC, Staudigel A, Salvenmoser S et al. Cross-resistance to medical and agricultural azole drugs in yeasts from the oropharynx of human immunodeficiency virus patients and from environmental Bavarian vine grapes. Antimicrob Agents Chemother 2007; 51 : 3014-6.

37 Verweij PE, Snelders E, Kema GH et al. Azole resistance in Aspergillus fumigatus: a side-effect of environmental fungicide use? Lancet Infect Dis 2009; 9: 789-95.

38 Faria-Ramos I, Tavares PR, Farinha $\mathrm{S}$ et al. Environmental azole fungicide, prochloraz, can induce cross-resistance to medical triazoles in Candida glabrata. FEMS Yeast Res 2014; 14: 1119-23.

39 Hube B. Fungal adaptation to the host environment. Curr Opin Microbiol 2009; 12: 347-9.

40 Berkow EL, Manigaba K, Parker JE et al. Multidrug transporters and alterations in sterol biosynthesis contribute to azole antifungal resistance in Candida parapsilosis. Antimicrob Agents Chemother 2015; 59: 5942-50.

41 Souza ACR, Fuchs BB, Pinhati HMS et al. Candida parapsilosis resistance to fluconazole: molecular mechanisms and in vivo impact in infected Galleria mellonella larvae. Antimicrob Agents Chemother 2015; 59: $6581-7$.

42 Kolaczkowski M, Kolaczkowska A, Motohashi N et al. New high-throughput screening assay to reveal similarities and differences in inhibitory sensitivities of multidrug ATP-binding cassette transporters. Antimicrob Agents Chemother 2009; 53: $1516-27$.

43 Silva AP, Miranda IM, Guida A et al. Transcriptional profiling of azole-resistant Candida parapsilosis strains. Antimicrob Agents Chemother 2011; 55: 3546-56.

44 Zhang $\mathrm{W}, \mathrm{Xu}$ J, Dong $\mathrm{F}$ et al. Effect of tetraconazole application on the soil microbial community. Environ Sci Pollut Res 2014; 21: 8323-32.

45 Brilhante RSN, Castelo Branco DSCM, Duarte GPS et al. Yeast microbiota of raptors: a possible tool for environmental monitoring. Environ Microbiol Rep 2012; 4: 189-93.

46 Brilhante RSN, de Aragão Rodrigues PH, de Alencar LP et al. Evidence of fluconazole-resistant Candida species in tortoises and sea turtles. Mycopathologia 2015; 180: 421-6.

47 Chowdhary A, Kathuria S, Xu J et al. Emergence of azole-resistant Aspergillus fumigatus strains due to agricultural azole use creates an increasing threat to human health. PLoS Pathog 2013; 9: 3-7.

48 Faria-Ramos I, Farinha S, Neves-Maia J et al. Development of crossresistance by Aspergillus fumigatus to clinical azoles following exposure to prochloraz, an agricultural azole. BMC Microbiol 2014; 14 155.

\section{Supporting information}

Additional supporting information may be found in the online version of this article.

Table S1. Primers used for gene expression analysis of Candida parapsilosis ATCC 22019 subjected to resistance induction assay through qPCR.

Table S2. Antifungal susceptibility of Candida parapsilosis ATCC 22019 independent replicates to fluconazole, itraconazole or voriconazole after resistance induction assay through the exposure to increasing concentrations of tetraconazole, malathion or fluconazole, during 7 weeks 